\title{
Effect of Micro-Dimple Aspect Ratio on Load-carrying Capacity of the Friction Pair
}

\author{
Kangmei $\mathrm{Li}^{1 \text {, a }}$, Jun $\mathrm{Hu}^{1, \mathrm{~b}, ~ *}$, Yang $\mathrm{Zhou}^{2, \mathrm{c}}$ \\ ${ }^{1}$ School of Mechanical Engineering, Donghua University, Shanghai201620, China \\ ${ }^{2}$ Engineering Department, Bombardier Sifang (Qingdao) Transportation Ltd., Qingdao266111, China \\ akmli@dhu.edu.cn, b hujun@dhu.edu.cn, ${ }^{\mathrm{a}}$ zhouyang@126.com \\ *Corresponding author
}

Keywords: micro-dimple array, aspect ratio, load-carrying capacity, CFD simulation

\begin{abstract}
Surface texturing is considered as an efficient way to improve tribological performance of friction pairs. The lubrication model of sliding surface with micro-dimple array was proposed by using the method of three-dimensional Computational Fluid Dynamics (CFD). The typical pressure distribution of the lubricant was investigated. It was found that, with proper parameters, the total pressure in the calculation domain could be positive, which is helpful for the increase of loadcarrying capacity. The effect of micro-dimple aspect ratio on load-carrying capacity was analyzed. It was found that the main mechanism for the improvement of load-carrying capacity is the comprehensive results of wedging effect and recirculation effect of the dimple. Therefore, there exists an optimum value of aspect ratio which leads to the best load-carrying capacity of microdimple unit.
\end{abstract}

\section{Introduction}

Surface texturing is one of the most promising solutions for improving load-carrying capacity of mechanical systems. The benefits of surface texturing and the effects of texturing parameters on load-carrying capacity were theoretically and experimentally investigated over the past two decades. Parametric studies were conducted for various applications such as thrust bearings [1-3], journal bearings [4], engine cylinders [5-7] and mechanical seals [8]. It was concluded that there are optimal texturing parameters with which the friction pair exhibits optimal load-carrying capacity.

In the last two decades, due to the fast development of computational techniques, the mechanisms for surface texturing to improve load-carrying capacity were numerically investigated. Based on twodimensional CFD method, tribological performances of surface with groove-shaped texture were studied. Sahlin et al. [9] compared the lubrication characteristics of groove texture with arc-shaped cross section against that with spline-shaped cross section. The effects of groove depth, groove width and Reynolds number on load-carrying capacity were studied. Shi et al. [10] developed a twodimensional CFD model to investigate the effects of cavitation pressure, sliding speed, sliding pitch angle and texture scale on fully lubricated sliding with cavitation. Ramesh et al. [11] solved the N-S equations by two-dimensional CFD and predicted the texture-induced lift.

From above studies, it can be seen that two-dimensional CFD technique is commonly used in simulation of groove texture. However, due to the geometrical complexity of micro-dimple array, the two-dimensional CFD is not able to simulate the interaction between the friction pair and the lubricant correctly. Han et al. [12] successfully proposed a three-dimensional CFD method to study the tribological characteristics of micro-dimple. The effects of micro-dimple size and Reynolds number on film pressure, friction force as well as friction coefficient were investigated.

It is noticed that the published papers about hydrodynamic lubrication of micro-dimple textured surface using three-dimensional CFD are very limited. In this study, the three-dimensional CFD method based on N-S equations is established. The main mechanism for the improvement of loadcarrying capacity by micro-dimple texturing is investigated and the optimum aspect ratio is discussed. 


\section{Mathematical Modeling}

The N-S equations which are the equations of momentum conservation considering inertia terms are used as the governing equations. At the same time, the behavior of lubricant in micro-dimple array satisfies the law of mass conservation.

In order to facilitate modeling and analysis, some assumptions were made as follows: a) The body force is considered negligible; b) No slip of lubricant is supposed to occur on the boundary, which means the velocity of lubricant close to friction pair surface is identical with that of friction pair surface; c) The lubricant is considered as Newtonian fluid and incompressible.

Based on above assumptions, the N-S equations could be simplified as[13]:

$$
\begin{gathered}
\rho\left(u \frac{\partial u}{\partial x}+v \frac{\partial u}{\partial y}+w \frac{\partial u}{\partial z}\right)=-\frac{\partial p}{\partial x}+\eta\left(\frac{\partial^{2} u}{\partial x^{2}}+\frac{\partial^{2} u}{\partial y^{2}}+\frac{\partial^{2} u}{\partial z^{2}}\right) \\
\rho\left(u \frac{\partial v}{\partial x}+v \frac{\partial v}{\partial y}+w \frac{\partial v}{\partial z}\right)=-\frac{\partial p}{\partial y}+\eta\left(\frac{\partial^{2} v}{\partial x^{2}}+\frac{\partial^{2} v}{\partial y^{2}}+\frac{\partial^{2} v}{\partial z^{2}}\right) \\
\rho\left(u \frac{\partial w}{\partial x}+v \frac{\partial w}{\partial y}+w \frac{\partial w}{\partial z}\right)=-\frac{\partial p}{\partial z}+\eta\left(\frac{\partial^{2} w}{\partial x^{2}}+\frac{\partial^{2} w}{\partial y^{2}}+\frac{\partial^{2} w}{\partial z^{2}}\right)
\end{gathered}
$$

Where $u, v$ and $w$ are the velocities of the fluid along $x, y, z$ axes, respectively; $\eta$ is dynamic viscosity; $p$ is pressure; $\rho$ is density of the fluid.

The simplified continuation equation could be expressed as:

$$
\frac{\partial u}{\partial x}+\frac{\partial v}{\partial y}+\frac{\partial w}{\partial z}=0
$$

In order to reduce the number of independent variables and make the simulation results more visualized, the dimensionless variables are defined as follows:

$$
\begin{aligned}
& x^{*}=x / l ; y^{*}=y / l ; z^{*}=z / h_{0} ; \\
& u^{*}=u / v_{0} ; v^{*}=v / v_{0} ; w^{*}=w /\left(v_{0} l / h_{0}\right) ; \\
& p^{*}=p / p_{0}
\end{aligned}
$$

Where $l$ is the characteristic length of micro-dimple unit, $v_{0}$ is the characteristic velocity of lubricant, $h_{0}$ is the thickness of lubricant film which is identical with the gap between the friction pair, $h$ is the depth of micro-dimple and $d$ is the diameter of micro-dimple (see Fig.1).

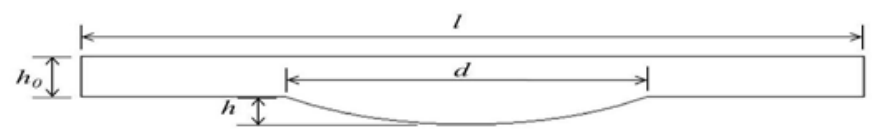

Figure 1. Sectional view of the fluid in micro-dimple unit

By substituting the above dimensionless variables into the simplified N-S equations, the N-S equations could be expressed as:

Along the direction of $\mathrm{x}$ axis:

$$
\begin{aligned}
& \frac{\rho v_{0}{ }^{2}}{l}\left(u^{*} \frac{\partial u^{*}}{\partial x^{*}}+v^{*} \frac{\partial u^{*}}{\partial y^{*}}+\left(\frac{l}{h_{0}}\right)^{2} w^{*} \frac{\partial u^{*}}{\partial z^{*}}\right) \\
& =-\frac{p_{0}}{l} \frac{\partial p^{*}}{\partial x^{*}}+\left(\frac{\eta v_{0} l}{h_{0}^{3}}\right) \frac{\partial^{2} u^{*}}{\partial z^{* 2}}+\left(\frac{\eta v_{0}}{l^{2}}\right)\left(\frac{\partial^{2} u^{*}}{\partial x^{*}}+\frac{\partial^{2} u^{*}}{\partial y^{* 2}}\right)
\end{aligned}
$$

By letting $p_{0}=\eta v_{0} l / h_{0}^{2}$ and defining the Reynolds number as $R e=\rho v_{0} h_{0} / \eta$, the above equation could be simplified as:

$$
\begin{aligned}
& R_{e}\left(\frac{h_{0}}{l}\right)\left(u^{*} \frac{\partial u^{*}}{\partial x^{*}}+v^{*} \frac{\partial u^{*}}{\partial y^{*}}+\left(\frac{l}{h_{0}}\right)^{2} w^{*} \frac{\partial u^{*}}{\partial z^{*}}\right) \\
& =-\frac{\partial p^{*}}{\partial x^{*}}+\left(\frac{l}{h_{0}}\right) \frac{\partial^{2} u^{*}}{\partial z^{* 2}}+\left(\frac{h_{0}}{l}\right)^{2}\left(\frac{\partial^{2} u^{*}}{\partial x^{*}}+\frac{\partial^{2} u^{*}}{\partial y^{* 2}}\right)
\end{aligned}
$$


Similarly, the equations along the directions of $\mathrm{y}$ axis and $\mathrm{z}$ axis could be obtained:

The dimensionless continuity equation could be expressed as:

$$
\begin{array}{r}
R_{e}\left(\frac{h_{0}}{l}\right)\left(u^{*} \frac{\partial v^{*}}{\partial x^{*}}+v^{*} \frac{\partial v^{*}}{\partial y^{*}}+\left(\frac{l}{h_{0}}\right)^{2} w^{*} \frac{\partial v^{*}}{\partial z^{*}}\right) \\
=-\frac{\partial p^{*}}{\partial x^{*}}+\left(\frac{l}{h_{0}}\right) \frac{\partial^{2} v^{*}}{\partial z^{2}}+\left(\frac{h_{0}}{l}\right)^{2}\left(\frac{\partial^{2} v^{*}}{\partial x^{*}}+\frac{\partial^{2} v^{*}}{\partial y^{* 2}}\right) \\
R_{e}\left(\frac{h_{0}}{l}\right)\left(u^{*} \frac{\partial w^{*}}{\partial x^{*}}+v^{*} \frac{\partial w^{*}}{\partial y^{*}}+\left(\frac{l}{h_{0}}\right)^{2} w^{*} \frac{\partial w^{*}}{\partial z^{*}}\right) \\
=-\frac{\partial p^{*}}{\partial x^{*}}+\frac{\partial^{2} w^{*}}{\partial z^{*}}+\left(\frac{h_{0}}{l}\right)^{2}\left(\frac{\partial^{2} w^{*}}{\partial x^{*}}+\frac{\partial^{2} w^{*}}{\partial y^{2}}\right)
\end{array}
$$

$$
\frac{\partial u^{*}}{\partial x^{*}}+\frac{\partial v^{*}}{\partial y^{*}}+\frac{\partial w^{*}}{\partial z^{*}}=0
$$

The equations from (5) to (8) could be used to describe the behavior of the fluid in micro-dimple unit under the condition of hydrodynamic lubrication.

\section{Numerical Simulation}

Fig.2 shows the three-dimensional geometric model of the fluid domain for single micro-dimple unit. Due to the symmetrical characteristic of each micro-dimple unit, only half of the micro-dimple unit is chosen as the calculation domain.

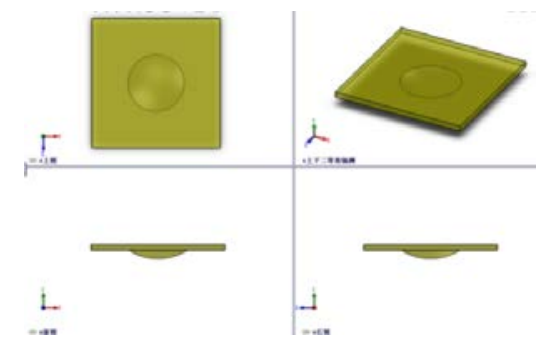

Figure 2. There-dimensional geometric model of single micro-dimple unit

Fig. 3 shows the typical meshed model of micro-dimple unit. The three-dimensional fluid domain was split into three domains. The micro-dimple was located in Domain Two and Domain Three. Due to the high concern of the entry, the exit and the interior of the dimple, the mesh density increased gradually from Domain One to Domain Three. The characteristic length of micro-dimple unit, the diameter and depth of micro-dimple are $2400 \mu \mathrm{m}, 1200 \mu \mathrm{m}$ and $20 \mu \mathrm{m}$, respectively. The film depth is $20 \mu \mathrm{m}$. The mesh information is summarized in Table I.

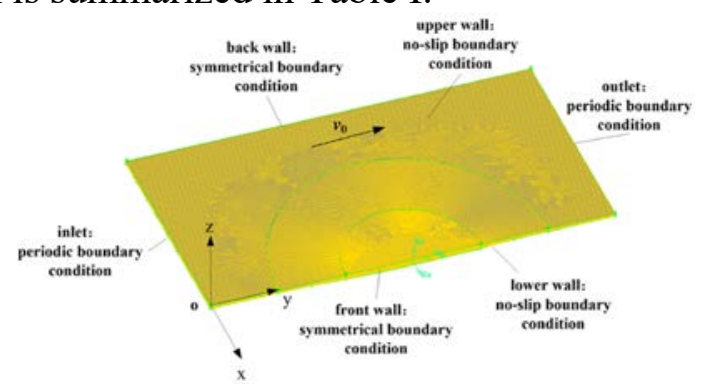

(a) Three-dimensional model of micro-dimple unit

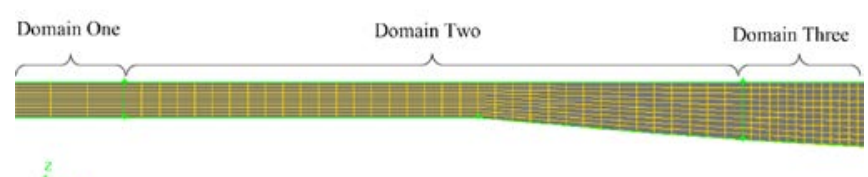

(b) Sectional view of the fluid in micro-dimple unit

Figure 3. The FEM model of micro-dimple unit 
TABLE I. The summary of mesh parameters

\begin{tabular}{|c|c|c|}
\hline Domain number & Average mesh size & Grid quantity \\
\hline Domain One & $6 \mu \mathrm{m}$ & 187610 \\
\hline Domain Two & $5 \mu \mathrm{m}$ & 126000 \\
\hline Domain Three & $4 \mu \mathrm{m}$ & 103060 \\
\hline Total & -- & 416670 \\
\hline
\end{tabular}

As shown in Fig.3, the upper and lower walls of the simulation model are set as no-slip boundaries. In order to simulate the relative movement of friction pair, the upper wall is moving along the positive direction of $\mathrm{y}$ axis with the velocity of $\mathrm{v} 0$ while the lower wall is assumed stationary. In addition, the inlet and outlet of the model along y axis are set as periodic boundary condition. The two walls along $\mathrm{x}$ axis are set as symmetrical boundary condition.

The model is calculated by using CFD software FLUENT with SIMPLE algorithm. The parameters used in simulation are summarized in Table II.

TABLE II. Parameters for cfd simulation

\begin{tabular}{|c|c|}
\hline Parameter & Value \\
\hline Texture density, $\rho_{t}$ & $5 \%, 13 \%, 20 \%, 50 \%$ \\
\hline Aspect ratio, $\lambda$ & $0.017,0.033,0.05,0.075,0.1$ \\
\hline Reynolds number, & $5,50,250$ \\
\hline
\end{tabular}

\section{Results and Discussion}

\subsection{Pressure Distribution of Micro-dimple Unit}

The typical pressure distribution in micro-dimple unit is shown in Fig.4. It is shown that when the lubricant flows from the inlet (point A) to the outlet of the calculation domain (point D), the pressure first decreases gradually to a minimum at the entry of the micro-dimple (point B). Then it starts to increase and reaches a maximum at the exit of the micro-dimple (point $C$ ). From point $C$ to point $D$, the pressure decreases to a value at point $\mathrm{D}$ which is the same as that at point $\mathrm{A}$.

The hydrodynamic pressure becomes positive in the convergent part of the gap while negative hydrodynamic pressure generates in the divergent part. It is found that with suitable parameters, the magnitude of positive pressure could be larger than that of negative pressure. As a result, the total integration of the pressure in the micro-dimple unit becomes positive. This phenomenon is related to the wedging effect of micro-dimple. The hydrodynamic pressure in micro-dimple unit offers extra carrying force which leads to the increase of load-carrying capacity.

\subsection{Effect of Aspect Ratio on Dimensionless Average Film Carrying Force}

The film carrying force is calculated by integrating the pressure on the upper wall, which could be expressed by (9):

$$
F_{z}=\iint p(x, y) \mathrm{dxdy}
$$

Where $F_{z}$ and $p(x, y)$ are the film carrying force and the pressure distribution, respectively. The dimensionless form of average film carrying force is given by (10):

$$
F_{z}^{*}=\frac{F_{z}}{p_{0} S_{t}}=\frac{F_{z} h_{0}^{2}}{S_{t} \eta v_{0} l}
$$




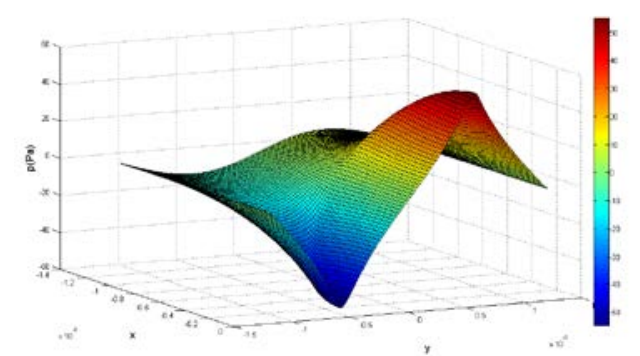

(a) 3D pressure distribution

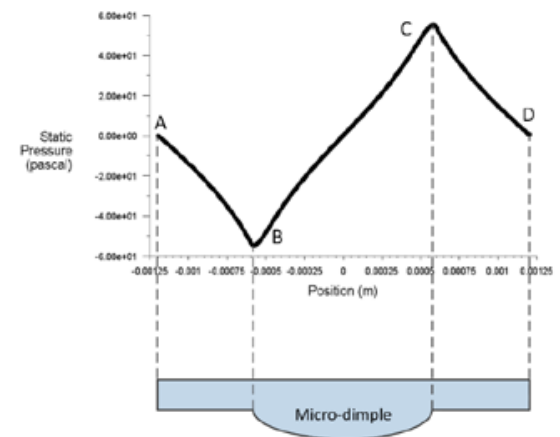

(b) 2D pressure distribution on middle section

Figure 5. Pressure distribution on upper wall of the lubricant in micro-dimple unit

Where $S_{t}$ is the area of upper wall of the calculation domain. It should be noted that large dimensionless average film carrying force indicates good load-carrying capacity of friction pair.

Fig.6 shows the effect of aspect ratio on dimensionless average film carrying force with various Reynolds numbers of 5, 50, and 250. (Texture density of 5\%, 13\%, 20\% and 50\% are abbreviated as tex $5 \%$, tex $13 \%$, tex $20 \%$ and tex $50 \%$, respectively).

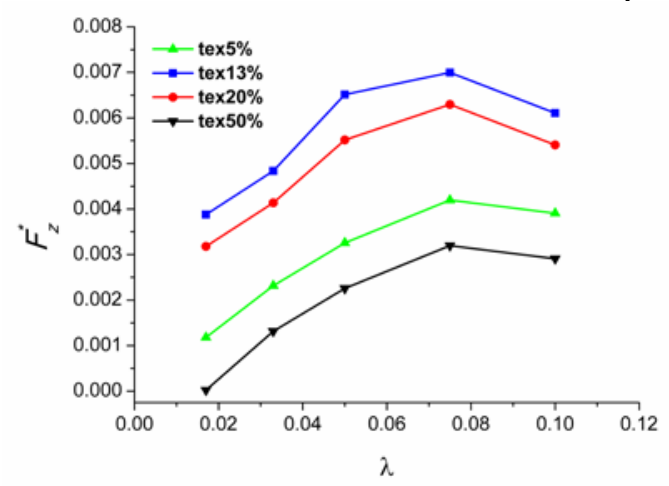

(a) $R_{e}=5$

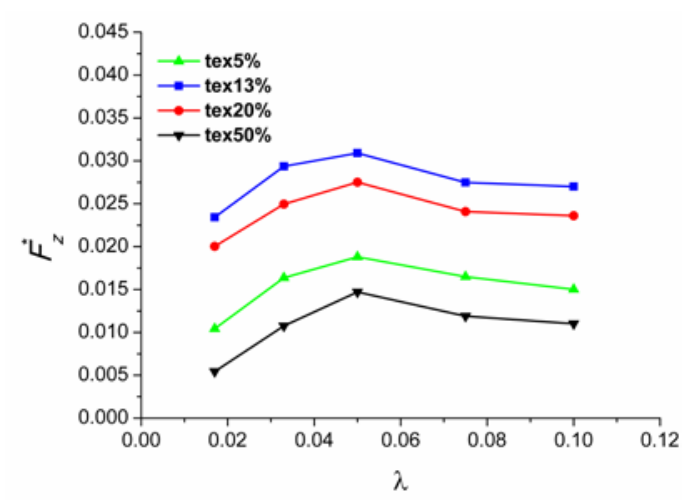

(b) $R_{e}=50$

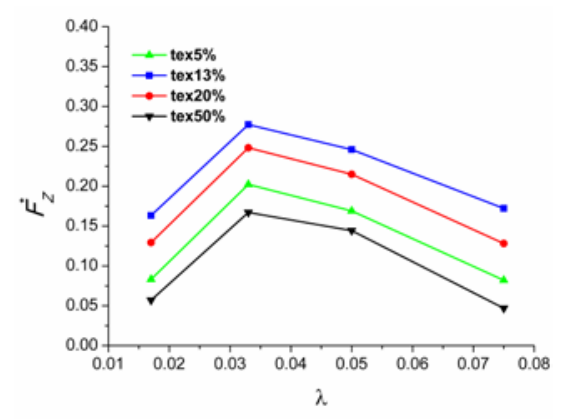

(c) $R_{e}=250$

Figure 6. Effect of aspect ratio on film carrying force

It was found that under the certain Reynolds number and texture density, there is an optimum aspect ratio at which the film carrying force reaches a maximum. Moreover, the optimum aspect ratio varies with the Reynolds number. 
In this study, the diameter of micro-dimple is set constant. The change of aspect ratio is made by changing micro-dimple depth. The inertia force increases with micro-dimple depth and enhances the wedging effect which is benefit to the improvement of load-carrying capacity. When the depth further increases to a certain value, however, the turbulence occurs and therefore load-carrying capacity is reduced due to energy dissipation. This phenomenon could be called recirculation effect. Because of the results of wedging effect and recirculation effect, there exists an optimum aspect ratio which leads to the best load-carrying capacity of micro-dimple unit.

\section{Conclusions}

Based on three-dimensional CFD simulation method, the load-carrying capacity of micro-dimple array under hydrodynamic lubrication was investigated. The main conclusions of this study are as follows:

(1) With proper aspect ratio, the total integration of the film pressure could be positive. As a result, the micro-dimple array could improve load-carrying capacity of friction pair;

(2) The reason for the improvement of load-carrying capacity of micro-dimple array is the comprehensive results of wedging effect and recirculation effect;

(3) For a certain Reynolds number, there exists the optimum aspect ratio with which the greatest load-carrying capacity under hydrodynamic lubrication is achieved.

\section{Acknowledgment}

The authors gratefully acknowledge the sponsorship from the National Natural Science Foundation of China (Grant No. 51605296), Shanghai Sailing Program (Grant No. 16YF1408300) and Young Teachers Training Program of Shanghai College (Grant No. ZZsl15029).

\section{References}

[1] V. Brizmer, Y. Kligerman and I. Etsion, "A laser surface textured parallel thrust bearing." Tribol. Trans. vol. 46, 2003, pp. 397-403, doi:10.1080/10402000308982643.

[2] I. Etsion, G. Halperin, V. Brizmer and Y. Kligerman, "Experimental investigation of laser surface textured parallel thrust bearings.” Tribol. Lett. vol. 179, 2004, pp. 295-300, doi:10.1023/B:TRIL.0000032467.88800.59.

[3] I. Etsion, "Improving tribological performance of mechanical components by laser surface texturing.” Tribol. Lett. vol. 17, 2004, pp. 733-737, doi:10.1007/s11249-004-8081-1.

[4] V. Brizmer and Y. Kligerman, “A laser surface textured journal bearing.” J. Tribo. vol. 134, 2012, p.031702, doi:10.1115/1.4006511.

[5] R. Golloch, G. R. Merker, U. Kessen and S. Brinkmann, "Functional properties of microstructured cylinder liner surfaces for internal combustion engines.” Lubr. Sci. vol. 11, 2005, pp. 307-324, doi:10.1002/tt.3020110403.

[6] G. Ryk, Y. Kligerman, I. Etsion and A. Shinkarenko, "Experimental investigation of partial laser surface texturing for piston-ring friction reduction.” Tribol. Trans. vol. 48, 2005, pp. 583-588, doi:10.1080/05698190500313544.

[7] B. Kim, Y. H. Chae and H. S. Choi, "Effects of surface texturing on the frictional behavior of cast iron surfaces.” Tribol. Int. vol. 70, 2014, pp. 128-135, doi:10.1016/j.triboint.2013.10.006.

[8] I. Etsion and L. Burstein, "A model for mechanical seals with regular microsurface structure.” Tribol. Trans. vol. 39, 1996, pp.677-683, doi:10.1080/10402009608983582.

[9] F. Sahlin, S. B. Glavatskih, T. Almqvist and R. Larsson, "2D CFD-analysis of micro-patterned surfaces in hydrodynamic lubrication.” J. Tribol. vol. 127, 2005, pp. 1657-1665, doi:10.1115/TRIB2004-64009. 
[10] X. Shi and T. Ni, "Effects of groove textures on fully lubricated sliding with cavitation.” Tribol. Int. vol. 44, 2011, pp. 2022-2028, doi:10.1016/j.triboint.2011.08.018.

[11] A. Ramesh, W. Akram, S. P. Mishra, A. H. Cannon, A. A. Polycarpou and W. P. King, "Friction characteristics of microtextured surfaces under mixed and hydrodynamic lubrication." Tribol. Int. vol. 57, 2013, pp. 170-176, doi:10.1016/j.triboint.2012.07.020.

[12] J. Han, L. Fang, J. Sun and S. Ge, "Hydrodynamic lubrication of microdimple textured surface using three-dimensional CFD.” Tribol. Tran. vol. 53, 2010, pp. 860-870, doi:10.1080/10402004.2010.496070.

[13] H. Versteeg and W. Malalasekera, “An introduction to computational fluid dynamics: the finite volume method.” Pearson, New Jersey,2007. 